Methods All patients referred to our tertiary institution for pancreatic intervention and successfully underwent SEMS were included.

Response (i.e. pain control) was assessed at 3 months by using a 1-10 visual analogue scale to compare pre- and post-intervention symptoms (a score $<2$ is complete, 3 or above is partial response).

Results A total of 15 patients (8 male and 7 female, mean age 52) received FC - SEMS 10 in main PD and 5 in the accessory duct (Santorini). 13 had previous endotherapy while for 2 patients this was the first attempt at endotherapy. 4 individuals with PD stones had ESWL prior to ERCP. Table 1 summarises the data on this patient group.

Placement of stents was feasible in all individuals. All had their stents removed at 4 months or earlier if they were symptomatic. One stent migrated proximally and associated with formation of an accessory duct abscess treated with antibiotics and stent removal. At 3 months, a total of 9 patients $(60 \%)$ reported complete resolution of pain whereas in another $2(13 \%)$ the response was parial. In the remaiing 4 individuals (27\%) there was no improvement after placement of FC-SEMS and those were subsequently removed; these individuals are considered for surgical drainage.

3 patients ( 2 complete responders and 1 partial responder) developed new strictures at the proximal end of the stents.

Conclusion Placement of FC-SEMS for treatment of BPS is feasible with an acceptable safety profile. Stent migration occured in one. New strictures seen in 3 patients and warrant further assessment. Future FC-SEMS designed for use in the pancreas may overcome this problem.

Disclosure of Interest None Declared.

\section{PTH-103 THE MANAGEMENT AND ASSESSMENT OF INCIDENTAL PANCREATIC CYSTS ON COMPUTER TOMOGRAPHY IN A NON-PANCREATIC CENTRE}

'S Kadir*, ${ }^{2} \mathrm{U}$ Sheikh, ${ }^{2} \mathrm{~J}$ Iqbal. 'Gastroenterology, Manchester Royal Informary, Manchester, UK; ${ }^{2}$ Gastroenterology, University Hospital South Manchester, Manchester, UK

\subsection{6/gutjnl-2014-307263.549}

Introduction With increased use and sensitivity of cross sectional imaging more incidental pancreatic cystic lesions are found. Studies have demonstrated a prevalence of $2.5 \%$ of cystic pancreatic lesions in asymptomatic patients on MRI and CT scanning. Cystic lesions have a wide variety of radiological appearances and prognostic outcomes. We aimed to review cases found to have incidental pancreatic cysts on CT scanning and ascertain the concordance between CT and EUS and the management of such lesions in a non-pancreatic centre.
Methods We retrospectively reviewed patients undergoing EUS for pancreatic cystic lesions found on incidentally on CT scan between 2010 and 2012. Twenty-five patients were included. Solid lesions with a cystic component were excluded.

Results There were 15 female; 10 male. Indications for CT scan included abdominal pain 32\%; weight loss 16\%; jaundice and suspected stone disease with abnormal USS $16 \%$. In all cases there was no preceding clinical suspicion of pancreatic cysts/disease. Median number of cysts was 1 (1-4); mean size $3 \mathrm{~cm}$ (1$11 \mathrm{~cm})$. In $70 \%$ of cases the cyst was $>3 \mathrm{~cm}$; and $<3 \mathrm{~cm}$ in $30 \%$. They were located in the HOP $36 \%$; BOP 32\%; TOP $16 \%$; NOP $4 \%$; multiple sites $12 \%$. The cyst was aspirated $+/-$ biopsy in $12(48 \%)$ cases, of which in $2(16 \%)$ this changed the CT diagnosis. Final diagnosis was pseudocyst 10 (40\%); IPMN 6 (24\%); simple cyst $2(8 \%)$; serous cystadenoma $2(8 \%)$; mucinous cystadenoma (MCA) 1 (4\%); cystic adenoca 1 (4\%); wegeners cyst 1 (4\%); renal cyst 1 (4\%).

In $32 \%$ (8 cases), the EUS findings were inconsistent with CT findings, due to IPMN and MCA in the vast majority. Greatest concordance between EUS and CT findings was in the diagnosis of pseudocysts.

Conclusion Increasing number of asymptomatic pancreatic cysts found incidentally will undoubtedly cause increased referrals to tertiary pancreatic centres. EUS is a better imaging modality with additional benefit of attaining samples. Performing EUS +/- cyst FNA provided an alternative diagnosis to CT in a significant percentage $(32 \%)$ of patients and helped streamline referrals for tertiary opinion. EUS should be considered in all patients presenting with incidental pancreatic cysts on cross-sectional imaging. This may be performed at the index hospital (if nonpancreatic centre) and should not be restricted to tertiary HPB centres if operator experience and confidence allows.

Disclosure of Interest None Declared.

\section{PTH-104 DIAGNOSTIC YIELD OF SECRETIN ENHANCED MRCP IN THE INVESTIGATION OF PATIENTS WITH ACALCULOUS PANCRAETICO-BILIARY TYPE ABDOMINAL PAIN}

SS Dronamraju*, J Scott, K Oppong, RM Charnley, M Nayar. Hepato-Biliary and Pancreas Unit, Freeman Hospital, Newcastle Upon Tyne, UK

\subsection{6/gutjnl-2014-307263.550}

Introduction Secretin enhanced magnetic resonance cholangiopancreatography (S-MRCP) has been used as part of the diagnostic algorithm for the diagnosis of patients presenting with acalculous biliary type abdominal pain (ABAP); the exact role of this diagnostic modality is unclear. The aim of this study was to assess the diagnostic yield of S-MRCP in a large HPB tertiary referral centre in the investigation of patients with ABAP.

\begin{tabular}{lll} 
Abstract PTH-104 Table 1 & Findings on S-MRCP in patients & who had a normal MRCP and / or a normal EUS \\
\hline S-MRCP findings & \multicolumn{1}{l}{ Patients with a normal MRCP } & $\mathbf{N}=37(\%)$ \\
\hline Normal & $29(78)$ & $\mathbf{N}=41(\%)$ \\
Obstruction at ampulla/ proximal PD & $5(13)$ & $19(46)$ \\
Cyst/IPMN & $1(3)$ & $12(28)$ \\
Divisum & $1(3)$ & $2(5)$ \\
Santoriniocele & - & $3(7)$ \\
PD stricture & $1(3)$ & $1(3)$ \\
Chronic pancreatitis & - & $2(5)$ \\
Poor quality scan & - & $1(3)$ \\
\hline
\end{tabular}


Methods Retrospective analysis of a prospectively maintained database of all patients presenting with ABAP in whom a S-MRCP was requested from June 2008 to May 2013. The findings of S-MRCP were compared with those of MRCP and EUS (performed prior to $S$ MRCP) and the diagnostic yield of $\mathrm{S}-\mathrm{MRCP}$ in the work up of a patient presenting with ABAP was estimated.

Results Of the 117 patients with ABAP [28 males and 89 female; mean age 48 yrs] were referred for S-MRCP, 114 (97.4\%) patients successfully completed the scan. Of these 37 patients who had a normal MRCP, S-MRCP identified significant findings in $8(22 \%)$ patients (Table 1$)$. In the present study 78 (67\%) patients had EUS. Out of the 41 patients who had a normal EUS, S-MRCP was able to identify significant pathology in 21 (54\%) patients (Table 1).

Conclusion This study suggests that S-MRCP has a 22 and 54\% additional diagnostic yield in ABAP patients who have a normal MRCP and a normal EUS respectively. The commonest abnormality identified in these patients on S-MRCP was obstruction at ampulla or proximal PD. S-MRCP should be considered in the diagnostic algorithm of patients with ABAP.

\section{REFERENCES}

1 Mariani A, Arcidiacono PG, Curioni $S$, et al. Diagnostic yield of ERCP and secretin-enhanced MRCP and EUS in patients with acute recurrent pancreatitis of unknown aetiology. Dig Liver Dis 2009 Oct;41(10):753-8

2 Matos C, Metens T, Devière J, et al. Pancreas divisum: evaluation with secretinenhanced magnetic resonance cholangiopancreatography. Gastrointest Endosc 2001 Jun;53(7):728-33

Disclosure of Interest None Declared.

\section{PTH-105 HOW OMINOUS IS THE "DOUBLE-DUCT" SIGN ?: A SINGLE CENTRE EXPERIENCE}

T Gardner*, K Padala, R Sinha, J Greenaway, D Joy. South Tees NHS Foundation Trust, Middlesbrough, UK

\subsection{6/gutinl-2014-307263.551}

Introduction "Double-duct" sign on ERCP (Endoscopic retrograde cholangio-pancreatogram) is considered suggestive of pancreatic or biliary malignancy. This sign is frequently encountered in radiological imaging. We wish to investigate the prognostic value of the "double-duct" sign in patients who undergo Magnetic resonance cholangio-pancreatography (MRCP), attempting to define the associated features which would predict underlying malignant disease. ${ }^{1,2}$

Methods A retrospective analysis of all the patients who underwent MRCP over a two year period; January 2011 to December 2012 was undertaken. All the radiological reports showing both a dilated common bile duct (CBD) and pancreatic duct (PD) or the "double-duct" sign were included. These were all interpreted and reported by specialist gastrointestinal radiologists. The demographics, liver biochemistry, final diagnosis and outcome for all patients with the "double-duct" sign were accessed using the radiology PACS ${ }^{\circledR}$ system, biochemical results WebICE®, hospital letters and case notes. Follow up information was available for a mean of 24months (range 12-36 months).

Results 1,367 patients underwent MRCP examination over two year period. 46 patients (3.5\% incidence) had "double-duct" sign (Table 1.) with a mean age of 69.5 years. The ratio of male to female patients was (M:F) 12:11. The commonest cause of "double-duct" sign was choledocholithiasis (29.4\%) followed by

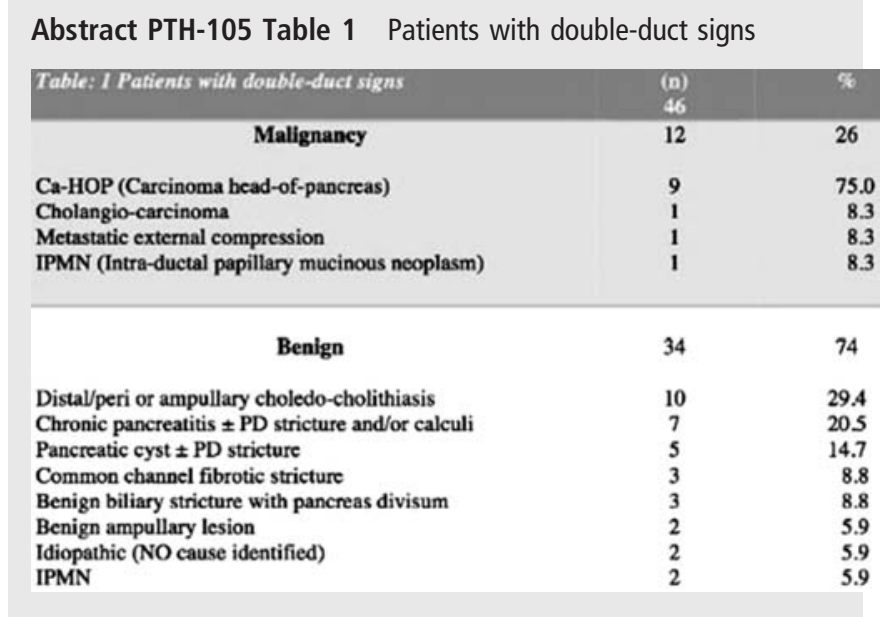

malignancy (26\%). Patients with jaundice in the context of "double-duct" sign had a higher incidence of malignancy (48\%). Nearly half of the patients, $(21 / 46 ; 46 \%)$ with "double-duct" sign were anicteric. None of the anicteric patients were found to have malignancy. Of the anicteric patients, 29\% (6/21) had completely normal liver test and the remaining 71\% (15/21) had some abnormality of the liver enzymes (raised GGT and/or Alkaline phosphatase). Three patients in the anicteric group had benign tumours ( 2 cases of benign IPMN and 1 case of benign ampullary tumour). The benign nature was confirmed on clinical and radiological follow-up. No surgical intervention was deemed appropriate for any of these patients. All three remained anicteric over the period of follow-up (13 months; unrelated death, 18 and 36 months respectively). Our results show that "double duct" sign in the absence of jaundice makes a malignant aetiology unlikely.

Conclusion In patients with cross-sectional imaging evidence of "double-duct" sign, the absence of jaundice makes a malignant aetiology unlikely. Conversely, in jaundiced patients a malignant cause is much more likely. Figures from larger series are needed to support this conclusion.

\section{REFERENCES}

1 Baillie J, et al. Biliary imaging: a review. Gastroenterology 2003; 125 (5):1565 2 Ahualli J. The double duct sign. Radiology2007;244 (1):314-5

Disclosure of Interest None Declared.

\section{Small bowel and nutrition}

\section{PTH-106 BILE ACID DIARRHOEA MASQUERADES AS DIARRHOEA-PREDOMINANT IRRITABLE BOWEL SYNDROME: RESULTS FROM A DUAL CENTRE PROSPECTIVE STUDY}

${ }^{1}$ I Aziz*, S Mumtaz, ${ }^{2} \mathrm{H}$ Bholah, ${ }^{3} \mathrm{FU}$ Chowdhury, ${ }^{1} \mathrm{DS}$ Sanders, ${ }^{2} \mathrm{AC}$ Ford. ${ }^{1}$ Department of Gastroenterology, Royal Hallamshire Hospital, Sheffield, UK; ${ }^{2}$ Leeds Gastroenterology Institute, Leeds, UK; ${ }^{3}$ Nuclear Medicine Department, St. James's University Hospital, Leeds, UK

\subsection{6/gutjnl-2014-307263.552}

Introduction Several studies have suggested that bile acid diarrhoea (BAD) can present with symptoms that are compatible with diarrhoea-predominant irritable bowel syndrome (IBS-D). However, uncertainty exists as these have often been 